\title{
Schmerzlinderung durch atemstimulierende Einreibung
}

\begin{abstract}
Unterschiedlichste Studien zeigten, dass $60-80 \%$ aller Bewohner von Langzeitpflegeeinrichtungen an Schmerzen leiden. Bettlägerigkeit, Inkontinenz und Schonhaltung sind unter anderem Gründe für die Schmerzzustände von Pflegeheimbewohnern. Weitere Untersuchungen haben ergeben, dass nur circa die Hälfe dieser Menschen eine adäquate Schmerztherapie erhält.
\end{abstract}

\Neben der medikamentösen Schmerztherapie gibt es zahlreiche pflegerische beziehungsweise nichtmedikamentöse Maßnahmen, die zur Schmerzlinderung beitragen können. In der praktischen Anwendung umfassen diese nicht-medikamentösen Maßnahmen zur Schmerzlinderung physikalische (peripher wirkende Maßnahmen), kognitiv-verhaltensorientierte Verfahren (zentral wirkende Maßnahmen) und körperliche Übungen. $\mathrm{Da}$ diese Interventionen größtenteils in der Pflege anzusiedeln sind, sollte auch „die Pflege“ immer wieder die Evidenz dieser Interventionen überprüfen und dafür Sorge tragen, dass diese Maßnahmen im Rahmen guter wissenschaftlicher Studien regelmäßig überprüft werden.

\section{Studienlage überzeugt nicht}

Für die atemstimulierende Einreibung (ASE), eine aus der basalen Stimulation stammende pflegerische schmerzlindernde Maßnahme, gab es nur wenige kleine Studien, die die Wirkung dieser Intervention überprüften. Dieses Ziel hatte die vorliegende Studie von Sladjana Romic et al. Sie sollte die Wirkung der ASE als pflegerische schmerzlindernde Maßnahme bei Bewohnern mit Schmerzproblematik nachweisen.

Fünf kognitiv nicht eingeschränkte Bewohner (drei Frauen, zwei Männer zwischen 80 und 97 Jahren) wurden ausgewählt und nahmen an der Studie teil, die in einem Zeitraum von sechs Monaten in einem geriatrischen Wiener Pflegekrankenhaus durchgeführt wurde. Drei unterschiedliche Assessmentmethoden wurden zur Einschätzung der Schmerzproblematik eingesetzt. Zur Selbsteinschätzung des Schmerzes nutzten die Untersucher ein Instrument, in dem eine numerische Rangskala (10-stufige Skala, auf der 0 „kein Schmerz" und 10 „stärkster vorstellbarer Schmerz" bedeutet) mit einer Smiley-Analogskala (bestand aus sechs Gesichtern, denen Zahlenwerte von 0-10 zugeordnet waren) und einer verbalen Ratingskala (bestand aus Adjektiven, z.B.: „kein Schmerz“, „schmerzt wenig“, „schmerzt extrem“, denen ebenfalls Zahlenwerte von 0-10 zugeordnet waren) kombiniert war.

\section{Kommentar}

Ziel dieses Projektes war es, so die Autoren der Studie, die Wirkung der atemstimulierenden Einreibung als schmerzlindernde pflegerische Maßnahme bei Bewohnern mit Schmerzproblematik nachzuweisen. Aufgrund der sehr geringen Fallzahl - fünf Probanden - und weiterer Limitationen der Studie (z.B. monozentrische Durchführung, Gelegenheitsstichprobe, fehlende Kontrollgruppe) wurde dieses Ziel jedoch nicht erreicht. Das Projekt wollte zu einer Professionalisierung des Pflegeberufs sowie in letztendlicher Konsequenz zu einer evidenzbasierten Versorgung von Schmerzpatienten beitragen. Dies ist allerdings in keinster Weise gelungen.

Nach einer Bewertung der vorliegenden Studie bleibt die direkte Wirkung von der ASE auf die Schmerzreduktion weiterhin unklar. Daher ist zu klären, ob es sich hier um eine Methode mit eher Kurzzeit- oder Langzeiteffekten handelt. Um die Evidenz dieser Intervention zu überprüfen bedarf es besser durchgeführter Studien mit einer größeren Fallzahl, einer Kontrollgruppe, einer Verblindung der Datenerheber und Datenauswerter und einer Randomisierung der Stichprobe. Damit dient die vorliegende Studie also eher zur Reflexion der Mitarbeiter über ihre Handlungen und Abläufe als zur Verallgemeinerung und zur Professionalisierung des Pflegeberufs.
Die Schmerzerfassung wurde über 14 Tage vor Start der Intervention, während der Intervention und über 14 Tage nach Abschluss der Intervention jeweils vor und nach der ASE im Rahmen der Körperpflege durchgeführt.

\section{Besser vor oder nach der} Körperpflege?

Zudem wurden die Pflegeberichte und extra für diese Studie durchgeführte Interaktionszeitgespräche ausgewertet. Dabei stand allerdings nicht die Schmerzlinderung, sondern das Wohlbefinden der Bewohner im Mittelpunkt. Die Auswertung zeigt vor Beginn der Intervention bei allen Teilnehmenden im Mittelwert niedrigere Schmerzangaben nach der Körperpflege als vor der Körperpflege. Während der Interventionsphase gibt es im Vergleich zum ersten Messzeitpunkt deutlich geringere Schmerzangaben, sowohl vor der Körperpflege als auch danach. Nach Ende der Interventionsphase (dritter Messzeitpunkt) gibt es deutlich niedrigere Werte als in den beiden anderen Vergleichszeiträumen. Allerdings zeigen die Mittelwerte zu diesem Zeitpunkt höhere Werte nach der Körperpflege als davor. Die Auswertung der Pflegeberichte und der Interaktionszeitgespräche lassen erkennen, dass sich bei der Mehrzahl der Bewohner die persönliche Einschätzung zu ihrem körperlichen Wohlbefinden durch die ASE verbesserte.

Quelle:

Romic, S., Dolhaniuk, I., Moser-Siegmeth, $V$. et al. Schmerzlinderung durch eine pflegerische Intervention in einem geriatrischen Pflegekrankenhaus. HeilberufeSCIENCE (2017) 8: 39-43

Autorenkontakt:

Referiert und kommentiert von

Oliver Ludwig, examinierter

Gesundheits- und

Krankenpfleger und

Diplompflegepädagoge, Jena.

E-Mail: oliver_ludwig@gmx.de 